\title{
KNOWLEDGE BASED SYSTEM FOR SATELLITE DATA SELECTION
}

\author{
Richa Goyal $^{\mathrm{a}}$, T. Jayasudha ${ }^{\mathrm{a}}$, Prateek Pandey ${ }^{\mathrm{b}}$, D. Rama Devi ${ }^{\mathrm{c}}$, A. Rebecca ${ }^{\mathrm{c}}$, M. Manju Sarma ${ }^{\mathrm{d}}$, B. Lakshmi \\ ${ }^{\text {a }}$ Software Group, SDAPSA, NRSC - (richagoyal, jayasudha t)@nrsc.gov.in \\ ${ }^{\mathrm{b}}$ Department of Computer Science and Engineering, CBIT - pprateek@ymail.com \\ ${ }^{c}$ NDC, SDAPSA, NRSC - (ramadevi_d, rebeccaswarna_a)@nrsc.gov.in \\ ${ }^{\mathrm{d}}$ Group Head, Software Group, SDAPSA, NRSC - manjusarma_s@nrsc.gov.in \\ ${ }^{\mathrm{e}}$ Deputy Director, SDAPSA, NRSC - lakshmi_b@nrsc.gov.in
}

KEY WORDS: Knowledge base, Satellite data product, Remote sensing applications

\begin{abstract}
:
In recent years, the use of satellite data for geospatial applications has multiplied and contributed significantly towards development of the society. Satellite data requirements, in terms of spatial and spectral resolution, periodicity of data, level of correction and other parameters, vary for different applications. For major applications, remote sensing data alone may not suffice and may require additional data like field data. An application user, even though being versatile in his application, may not know which satellite data is best suited for his application, how to use the data and what information can be derived from the data. Remote sensing domain experts have the proficiency of using appropriate data for remote sensing applications.

Entrenching domain expertise into the system and building a knowledge base system for satellite data product selection is vital. Non specialist data users need a user-friendly software which guides them to the most suitable satellite data product on the basis of their application. Such tool will aid the usage for apt remote sensed data for various sectors of application users. Additionally, the consumers will be less concerned about the technical particulars of the platforms that provide satellite data, instead focusing on the content and values in the data product, meeting the timelines and ease of access. Embedding knowledge is a popular and effective means of increasing the power of using a system. This paper describes a system, driven by the built-in knowledge of domain experts, for satellite data products selection for geospatial applications.
\end{abstract}

\section{INTRODUCTION}

The Indian remote sensing satellite system has one of the largest constellations of remote sensing satellites in operation today. These satellites provide data with varying spatial, spectral and temporal resolutions. The data acquired and dumped at ground stations is then processed to generate usable remote-sensing data products. The parameters while data product generation like level of processing and enhancement, projection and resampling characterize the end product and vary with its intended use.

Major remote sensing applications include agriculture, forestry, water resources, snow and glaciers, geology, cartography, coastal zone, marine fisheries, weather forecasting, ocean state forecasting, besides disaster monitoring and mitigation. Spatial, spectral and temporal resolution requirements of different applications are different. Regional vegetation mapping require very high spatial resolution data. Crop identification requires properly placed spectral bands in the red, near infrared and middle infrared wavelength regions. For some applications remote sensing data alone is not sufficient, and requires additional information. This application specific knowledge of suitability of data product and its usage is available with the domain experts.

The existing data search is based on resolution and is helpful to the users who have knowledge on remote sensing and data products. Application specific selection of remote sensing products requires skill and domain expertise. Users having knowledge about their application area but lacking knowledge about satellites and sensors get confused in choosing the appropriate data products and face many problems. To help the users in selecting an appropriate satellite data product depending on their application without the knowledge about satellite and sensors, a solution is designed and implemented as a web-based application, so as to help the user to navigate and obtain the precise satellite data product suitable for his application studies.

\section{ARCHITECTURE}

Knowledge Based System for Satellite Data Selection is essentially composed of three components: knowledge base, inference engine and leaning module [Figure 1].

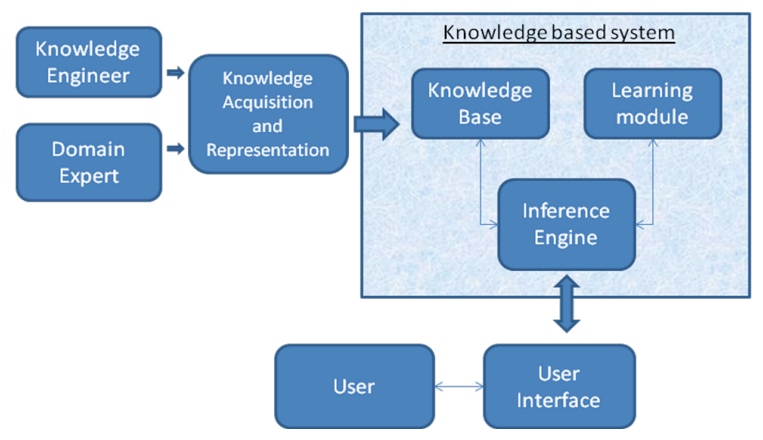

Figure 1: Architecture

The knowledge base represents the facts about the real world. It comprises of the known static data like satellites and sensors, gathered data from domain experts regarding study parameters and product preferences and dynamic usage pattern data. The inference engine is an automated reasoning system that evaluates the current state of the 
knowledge-base, applies relevant rules, arrives at a particular conclusion and also, asserts new knowledge into the knowledge base. The learning module is designed to capture the software usage pattern and subsequently refine application specific satellite product suitability. Additionally, it allows for dynamic inclusion of applications and study areas not built within the application.

\section{MODELLING AND IMPLEMENTATION}

\subsection{Gathering knowledge}

Acquiring, organizing and consolidating the knowledge from domain experts to support knowledge base systems is one of the key activities in building knowledge base systems. Knowledge acquisition was done by interviewing domain experts from various applications like Water, Urban and Agriculture. Application hierarchy comprising themes and subthemes was established from the initial information gathered. Further, application specific information was collected and the study specific parameters were indentified which determine the resolution and product suitability. This information was consolidated to arrive at the constants and variables present to build the application. The static data pertaining to applications and themes, study area repository, satellites and sensors and available set of resolutions and products was represented as facts. The rules were formulated from the dependencies determined between the study parameters and product specifications that drive the decision making ability of the software.

\subsection{Inference Engine}

The inference engine is the control structure that produces the output based on the data currently existing in the knowledge base and the rules of the expert system. It takes the input from the user and reaches logical outcomes based on the premises of the data and matching rule to deduce the result. It tries to derive new information about a given problem using the rules in the rule base and the situation-specific knowledge present in the working memory. Forward chaining mechanism [Figure 2] has been selected to implement the inference engine rules. Forward chaining takes the available data as input, searches a rule in the inference engine until it finds one where the antecedent matches the input data and then deduces the conclusion, resulting in the addition of new information to its dataset.

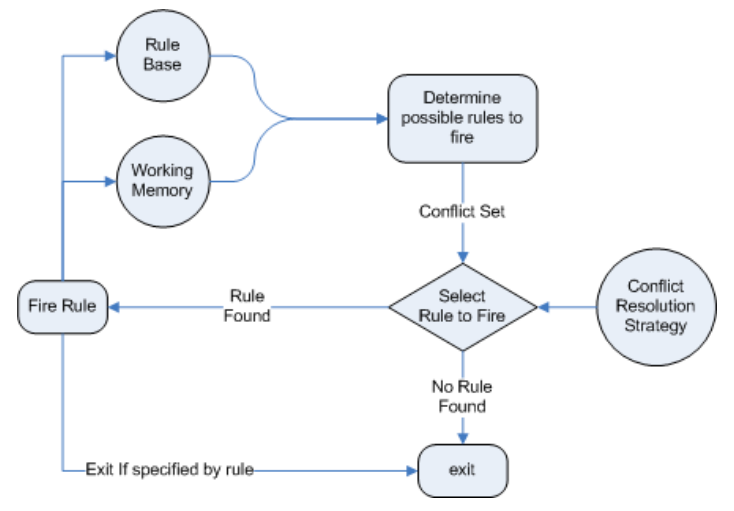

In order to implement rules for modelling the knowledge base, JESS a java expert system shell is used. JESS is a rulebased language for developing expert systems and can be seamlessly integrated with Java. JESS was originally inspired by the CLIPS expert system shell, but has grown into a complete, distinct Java-influenced environment of its own. JESS built applications have the capacity to "reason" using knowledge supplied in the form of declarative rules. For matching of rules to facts, JESS makes use of RETE algorithm. The Rete algorithm is a pattern matching algorithm for implementing production rule systems. It is used to determine which of the system's rules should fire based on its data store.

\subsection{Writing facts and rules}

Jess rules work by pattern-matching on facts. The facts are formulated from the static data present like satellites, sensors, resolutions, products, applications and study area. To add facts, a template is created with the help of "deftemplate". Various templates are created in this expert system, one such is shown below:

(deftemplate theme "Theme information" (slottheme_name) ; Name of the theme )

After creating templates the process of facts formation and adding it to working memory is done. Various facts are created in this expert system. Some of them are presented below:

\section{FACT 1:}

(assert (theme(theme_name Urban)))

\section{FACT 2:}

(assert (theme(theme_name Water))

(subtheme(subtheme_name \"Water bodies\"))

(applications(app_name \"Reservoirs \")))

\section{FACT 3:}

(assert (app_resolution (theme Water) (subtheme \"Glacierl") (app \"Glacier mapping \")))

\section{FACT 4:}

(assert (product type (resolution 2.5m) (start_year 2007) (end_year 2009)) (expertise_level(level Novice)))

The Rules are written in Java Expert System Shell (JESS) rule format. Depending upon the option selected by the users at user interface, facts are asserted, which will trigger the appropriate rules. Jess fires all the rules continuously when it's left-hand-side is satisfied and consequently, the 'then' part can be concluded All Jess rules are defined using the "defrule" construct. Rules are uniquely identified by their name. The name is followed by an optional documentation string that describes the purpose of the rule. The symbol $\Rightarrow$ (an equals sign followed by a greater-than sign) separates the rule's lefthand side (LHS, or if part) from its right-hand side (RHS, or then part). The symbol $=>$ can thus be read as then. This expert system contains 200 rules. Some of the rules are presented below:

Figure 2: Forward Chaining 
RULE 1:

(defrule theme-name "Rule to display theme names" $\Rightarrow$ (store THEMENAME “Agriculture, Urban, Water”)

\section{RULE 2:}

(defrule Agriculture-subthemenames "Rule to display names of the subtheme under Agriculture theme" (theme (theme_name Agriculture))

(store SUBTHEME \“Crop condition assessment, Site suitability analysis, Crop inventory〉”)

)

\section{RULE 3:}

(defrule U-Env-Appnames "Rule to display names of the applications under Urban theme and Environment subtheme")

(theme (theme_name Urban))(subtheme (subtheme_name Environment))

$$
\begin{aligned}
& \Rightarrow \\
& \text { (store APPNAME \"Vegetation, Water bodies } \backslash ")
\end{aligned}
$$

RULE 4:

(defrule U PP Small1 \"Rule to display resolution with pop $<100000$ for Plan Preparation under Urban $\backslash "$ "

( app_resolution (theme Urban) (subtheme ।"Plan preparation $\backslash ")$ (size-pop ?p-value\&:(< ?p-value 100000)) (app \"Mater/development plan\"))"

$$
=>
$$

(store RES VALUE1 \"High Resolution 1m, Multispectral data achievable on 1:6,000 scale which is available every 125 days $\backslash "))$

\section{RULE 5:}

(defrule 23m_Expert2 $\backslash$ "Rule to display suitable product type for $23 \mathrm{~m}$ resolution with expertise level as Expert $"$ " (product_type (resolution 23m) (start_year?st_yr\&:(>

\subsection{Building the Graphical user interface}

The graphical user interface provides a medium to the user to interact with the software. This interface has been designed keeping in mind the user-base and the aim of the software. It has been designed to accept the inputs from the user pertaining to the details of his remote sensing application and study. The inputs captured by the software system residing at the client are then sent to the server which in-turn passes them to the inference engine. The conclusion drawn from inputs are then formulated into outputs and displayed to the user.

The GUI for Knowledge Based System for Satellite Data selection is developed using Adobe Flex, a cross-platform rich internet applications (RIA) technology for development of web-based applications, supported by Apache Tomcat as web-server and Java and Jess based backend. A common interface has been designed to suffice all the applications and studies and supports easy modifications and appending new study areas and satellite data product options.

\subsection{Learning Module}

The learning module is the component that comes into play with the usage of the software. The elementary version of the software has been designed taking the inputs from the domain experts. However, the user's selection of satellite data product for his study is also an important piece of information. This information is captured and designed to eventually refine the initial set of decision making rules after validation.

In the scenario where a user wants information for a certain area of study that is not included within the software, provision for inclusion of the same has been designed. The information for the required application and study will be collected from the domain expert and subsequently built into the system.

\section{RESULTS}

The following figures present some samples from the proposed software.

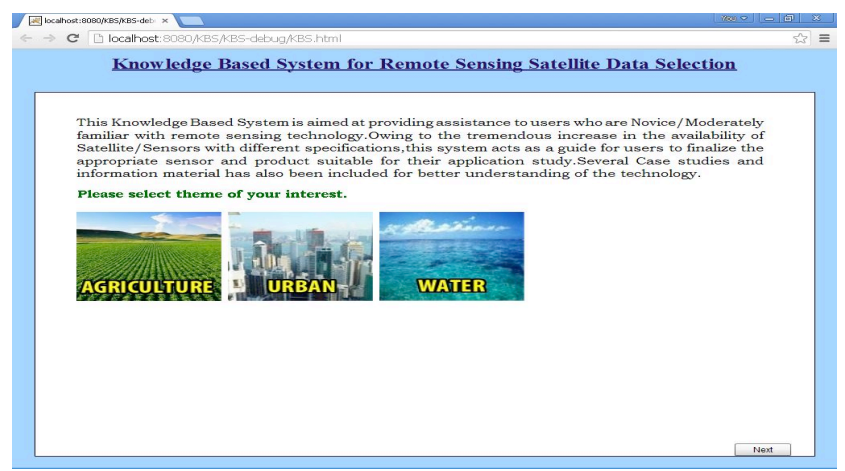

Figure 3: Home page

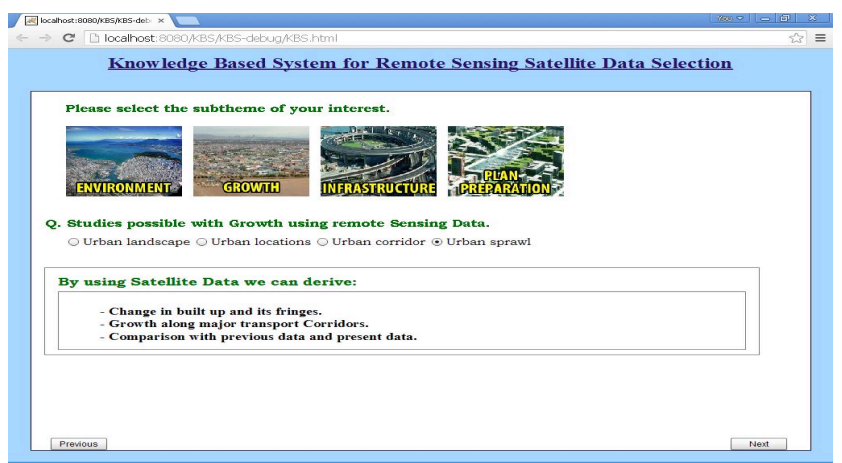

Figure 4: Application selection page

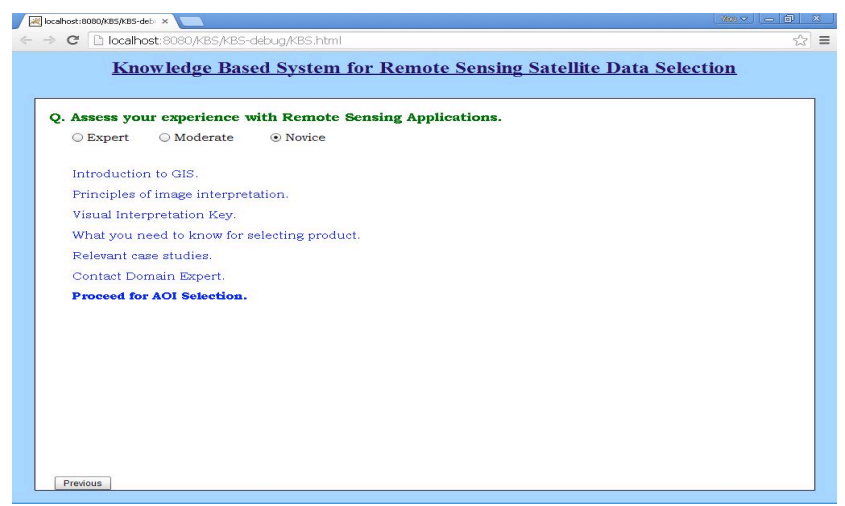

Figure 5: Expertise level selection page 


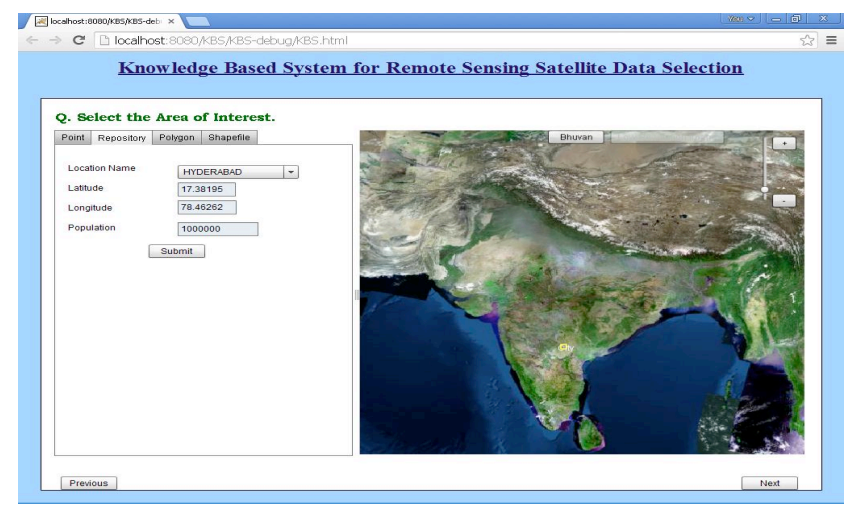

Figure 6: Area of location selection page

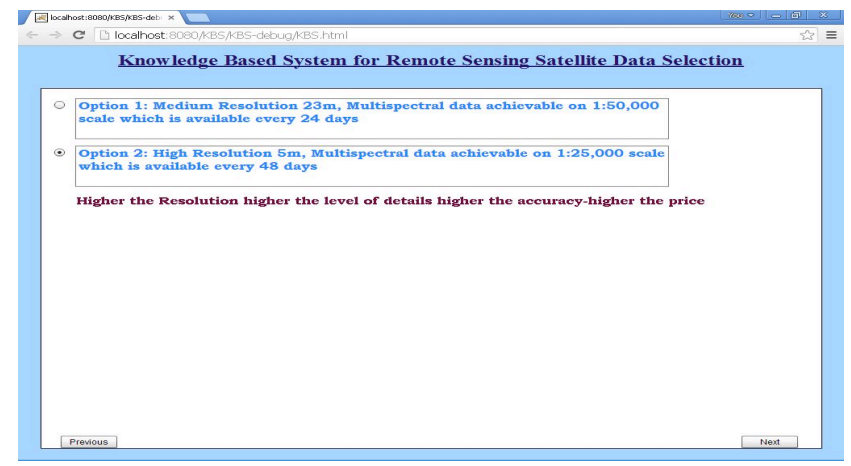

Figure 7: Resolution selection page

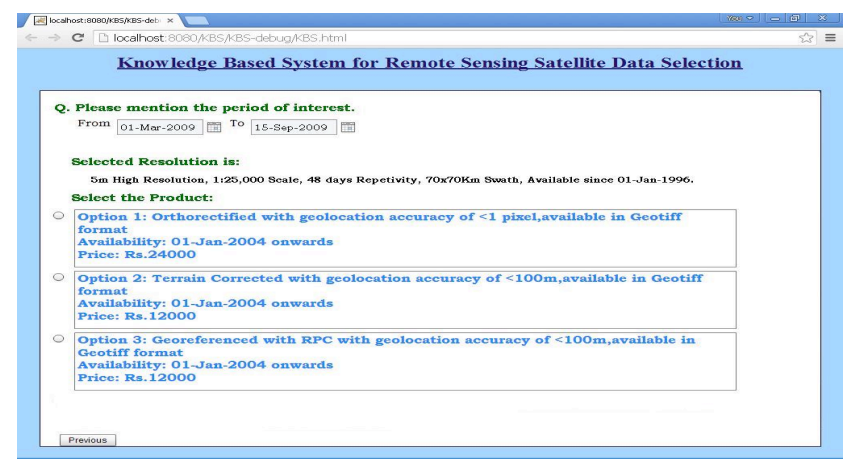

Figure 8: Satellite data product selection page

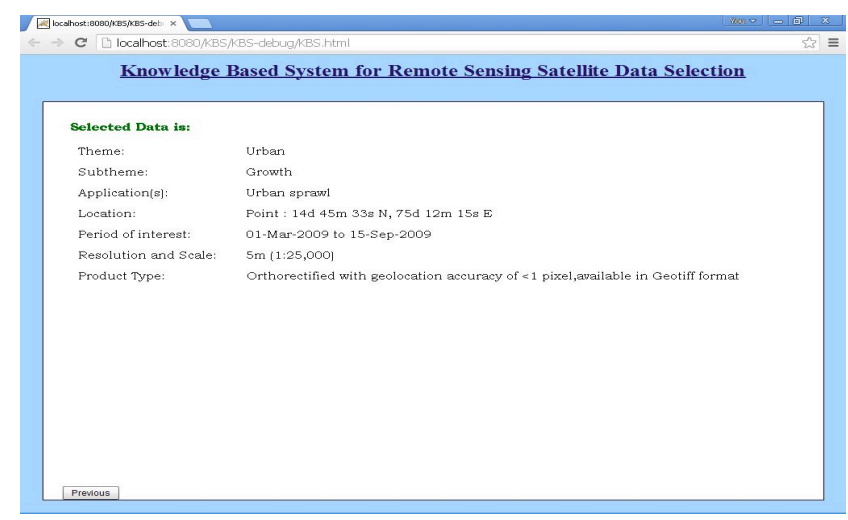

Figure 9: Selection Summary

\section{CONCLUSIONS AND FUTURE WORK}

The expert system developed here fulfils the purpose of guiding a user, irrespective of his cognition, about satellites and sensors, to identify the best suitable satellite-data product for his application study. Information gathered from domain experts are formulated in the form of facts and rules and depending on the input given by the users, the appropriate rule is invoked from the knowledge base, thereby providing the best suitable satellite data-product for his application study.

Presently this system contains three applications, Agriculture, Water and Urban. Extension of this system includes appending more of such remote sensing applications and corresponding information gathered from respective domain experts. Also, after the acceptance and validation of the software by the users, it shall be integrated with the existing data selection and ordering system.

\section{ACKNOWLEDGEMENT}

The authors would like to thank Director, NRSC for giving us the opportunity to realize the product. The authors are grateful to the application domain experts for initially providing information that is the base of this software, and for providing critical review to improve the product to meet the purpose for which it was envisaged. The authors would also like to acknowledge each and every individual in data processing area and NRSC data centre for their support.

\section{REFRENCES}

[1]. Patterson, D. W. "Introduction to Artificial Intelligence and Expert Systems", Prentice-Hall of India Private Limited, 1990. pp. 326-337.

[2]. George F. Luger and William, A. Stubblefield, "Artificial Intelligence and the Design of Expert Systems", The Benjamin/ Cummings publishing Co., Inc., 1989.

[3]. Peter Jackson," Introduction to Expert Systems", Addison-WesleyLongman Publishing Co, 1999.

[4]. John Durkin,"Expert Systems: Design and Development",Macmillan publishers, 1994.

[5]. Ernest Friedman-Hill, Jess in Action: Java RuleBased Systems, Manning Publications, 2003.

[6]. Arun N Nambiar, Anish.K. Dutta, ,Expert System for Student advising using JESS" International Conference on Education and Information Technology, Pp. V1 312- V1 315, 2010.

[7]. Khumukcham R., Shikhar Kr. S, "JESS Based Expert System Architecture For Diagnosis Of Rice Plant Diseases: Design And Prototype Development", 2013 4th International Conference on Intelligent Systems, Modeling and Simulation, P 674-676, 2013.

[8]. Ken KaLun Ho and Meiliu Lu. "Web-based expert system for class schedule planning using JESS". In Proceedings of the 2005 IEEE International Conference on Information Reuse and Integration, pages 166-171. IEEE, 2005.

[9]. JESS, the Rule Engine for the JavaTM Platform. http://herzberg.ca.sandia.gov.

[10]. http://www.jessrules.com/jess/docs/71/basics.html 